\title{
Anisotropy of Magnetization Reversal and Magnetoresistance in Square Arrays of Permalloy Nano-Rings
}

\author{
A. V. Goncharov ${ }^{1}$, A. A. Zhukov ${ }^{1}$, V. V. Metlushko ${ }^{2}$, G. Bordignon ${ }^{3}$, H. Fangohr ${ }^{3}$, C. H. de Groot $^{4}$, J. Unguris ${ }^{5}$, \\ W. C. Uhlig ${ }^{5}$, G. Karapetrov ${ }^{6}$, B. Ilic ${ }^{7}$, and P. A. J. de Groot ${ }^{1}$ \\ ${ }^{1}$ School of Physics and Astronomy, University of Southampton, SO17 1BJ, U.K. \\ ${ }^{2}$ Department of Electrical and Computer Engineering, University of Illinois at Chicago, Chicago, IL 60607-0024 USA \\ ${ }^{3}$ School of Engineering Sciences, University of Southampton, SO17 1BJ, U.K. \\ ${ }^{4}$ School of Electronics and Computer Science, University of Southampton, SO17 1BJ, U.K. \\ ${ }^{5}$ National Institute of Standards and Technology, Gaithersburg, MD 20899-8412 USA \\ ${ }^{6}$ Materials Science Division, Argonne National Laboratory, Argonne, IL60439 USA \\ ${ }^{7}$ Cornell Nanofabrication Facility and School of Applied and Engineering Physics, Cornell University, Ithaca, NY 14853 USA
}

Magnetization reversal mechanisms and the impact of magnetization direction are studied in square arrays of interconnected circular permalloy nanorings using magnetooptical Kerr effect, local imaging, numerical simulations, and transport techniques.

Index Terms-Magnetic nano-structures, magnetization reversal, magnetoresistance, nano-rings.

\section{INTRODUCTION}

$\mathbf{M}$ AGNETIC nano-structures demonstrate a wealth of exciting properties, which are extremely sensitive to the shape and order of nano-elements interacting via exchange and dipolar coupling [1]. From an application point of view, such materials are important to the magnetic recording industry and, in particular, for rapidly developing magnetic random access memory (MRAM) devices [2]. Magnetic nano-rings are among the most promising contenders for the future MRAM applications [3]. The presence of a hole in the center of the ring eliminates the highly energetic vortex core existing in a dot element and constitutes an important advantage of ring geometry. Magnetic properties of separate nano-rings have been reported in several publications (e. g., [4]-[6], [12]). In this work we study square arrays of connected circular permalloy rings to explore magnetization reversal and magnetoresistance mechanisms in dependence on the magnetic field direction.

\section{EXPERIMENTAL PROCEDURE}

Periodic square arrays of $25 \mathrm{~nm}$ thick permalloy nano-rings (Fig. 1) have been prepared by e-beam lithography on a (100) silicon wafer spin-coated with bi-layer resists (PMMA and P(MMA-MAA) copolymer). Using e-beam evaporation in a high vacuum a permalloy film was deposited, and after a lift-off in acetone the arrays of magnetic permalloy rings were obtained. In this work we study square arrays with a period of $1000 \mathrm{~nm}$ of connected circular rings having external and internal diameters of 1100 and $650 \mathrm{~nm}$, respectively. Magnetooptical Kerr effect (MOKE) magnetometry, magnetoresistance, scanning electron microscopy with polarization

Digital Object Identifier 10.1109/TMAG.2006.878429

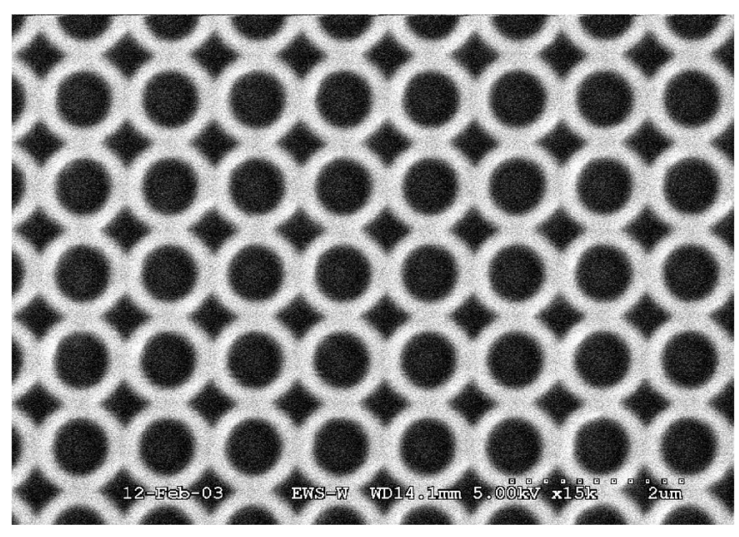

Fig. 1. SEM image of a permalloy array with interconnected circular nano-rings.

analysis (SEMPA) [7], and magnetic force microscopy (MFM) were used for characterization. Measurements of $M-H$ curves were performed at room temperature for magnetic fields in the film plane with various angles between the field direction and an edge of the square array. The transport measurements were made by a four probe method for different in-plane and out-off-plane magnetic field directions. Magnetic simulations have been made using the OOMMF software suite [8].

\section{RESULTS and DISCUSSION}

\section{A. In-Plane Magnetization Reversal Anisotropy}

For magnetic field directions close to the main axis of the square array we observe sharp one step magnetization switching [Fig. 2(a)]. More complicated behavior with a gradual magnetization rotation is observed near the $45^{\circ}$ type directions [Fig. 2(b)]. As can be seen from Fig. 3 the angular dependence of in-plane coercive fields demonstrates significant variations. Close to the main directions of square arrays $\left(\varphi=0^{\circ}\right.$ and 

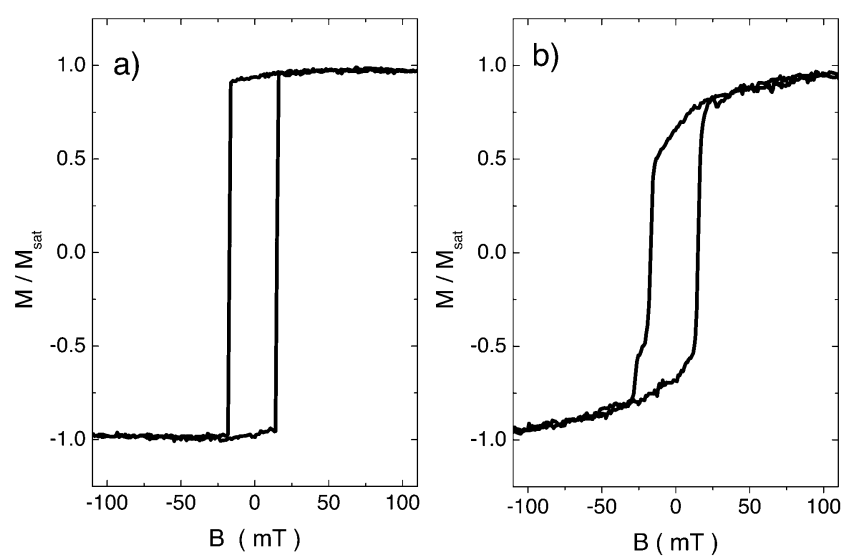

Fig. 2. MOKE magnetization loops for (a) $\varphi=0^{\circ}$ and (b) $\varphi=45^{\circ}$. The $\varphi=0$ direction corresponds to the magnetic field along an edge of a square lattice.

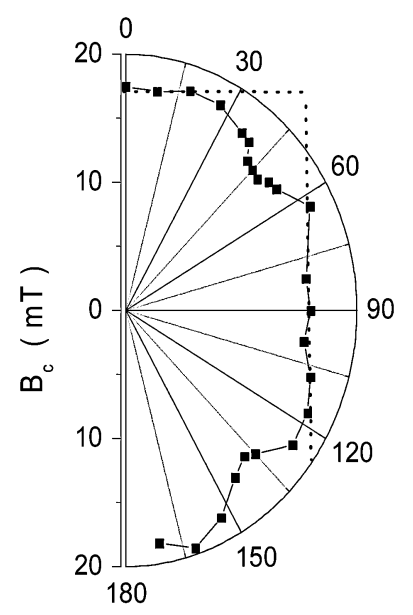

Fig. 3. Angular dependence of coercivity on the direction of the in-plane applied magnetic field. Dashed lines correspond to a fixed projection onto the main directions.

$90^{\circ}$ ) the coercive field roughly follows a fixed projection of magnetic field on the corresponding main direction. Near the $45^{\circ}$ type directions this behavior changes and a pronounced minimum is reached.

The origin of the angular anisotropy observed in our experiment is clearly related to the connections between rings, which break the circular symmetry. For the soft permalloy the material anisotropy is a minor factor and the coercive field should be determined by the interplay of dipolar and exchange interactions. In a separate ring an onion state is realized in the remanent state [4]-[6], [12]. The suppression of magnetostatic energy for the core of the onion state defines its preference for the alignment along the ring connections. This differs from square rings where the shape related dipolar term favors the 45 degree direction [9].

Our numerical simulations qualitatively reproduce the experimental behavior. The remanent state according to this analysis is an onion state [Fig. 4(a)] and the reversal mechanisms are realized by $90^{\circ}$ and $180^{\circ}$ rotations. Our SEMPA and MFM imaging confirms the presence of the onion state in connected square ring arrays [Fig. 4(a) and (c)]. A clear rhombic like domain can be
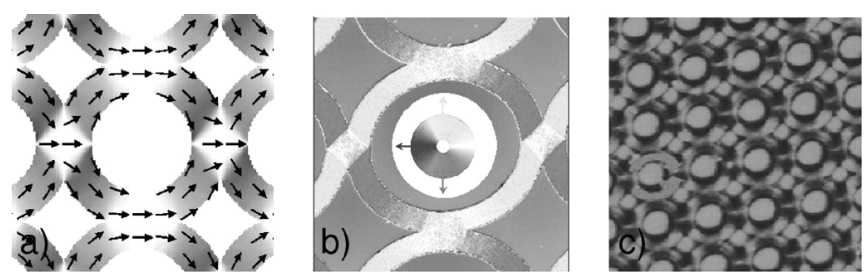

Fig. 4. Moment distribution from (a) numerical simulations, (b) SEMPA, and (c) MFM for the remanent state after field applied along the $\mathrm{x}$-axis.
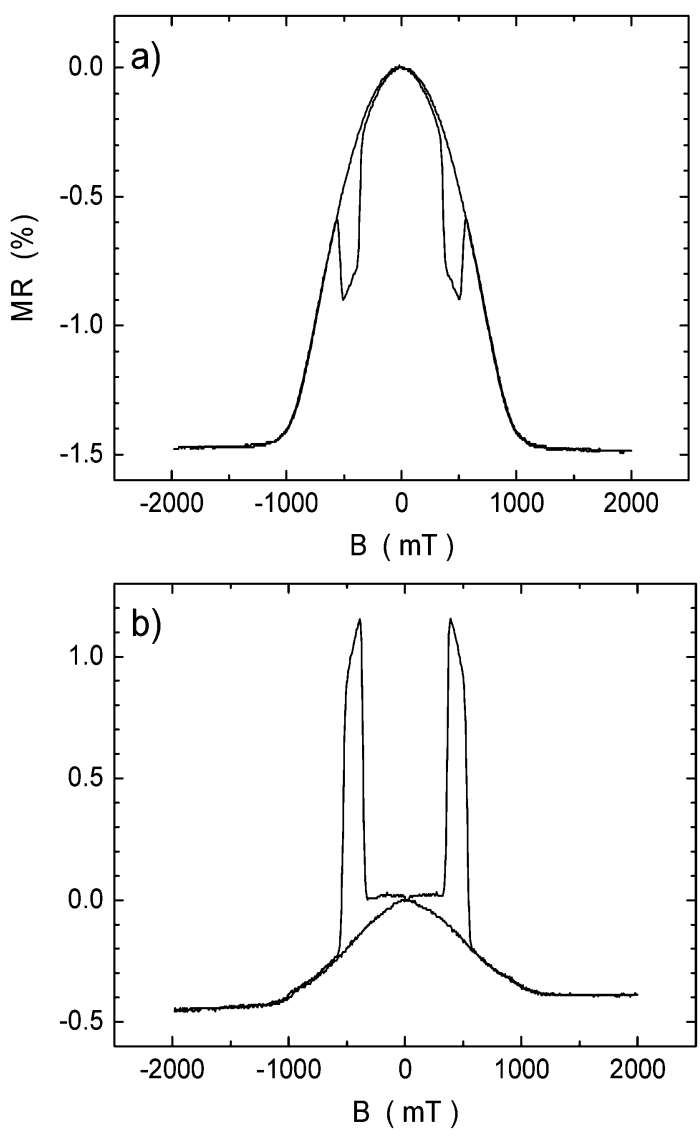

Fig. 5. Magnetoresistance of the interconnected circular ring array (Fig. 1) for $\mathrm{H}_{/ /}$component (a) parallel and (b) transverse to the electric current (directed along the edge of the square array).

seen at interconnections between the onion states. Such magnetic structures appear similar to observations in chains of circular Co rings [10].

\section{B. Magnetoresistance for Different In-Plane and Out-of-Plane Magnetic Field Directions}

For magnetic fields parallel to the film plane the magnetoresistance $(M R=R(H) / R(0)-1)$ demonstrate an anisotropic magnetoresistance (AMR) peak at small fields. For magnetic fields tilted out-of-plane two sharp peaks are superimposed on a characteristic $\sim H^{2}$ AMR behavior. As can be seen from Fig. 5 the sign of these peaks is dependent on the direction of the in-plane magnetic field component $\mathrm{H}_{/ /}$. It is negative for $\mathrm{H}_{/ /}$ parallel to the electric current and positive for the transverse orientation.

Using a rotational stage with high angular resolution, the magnetoresistance curves have been measured for various 


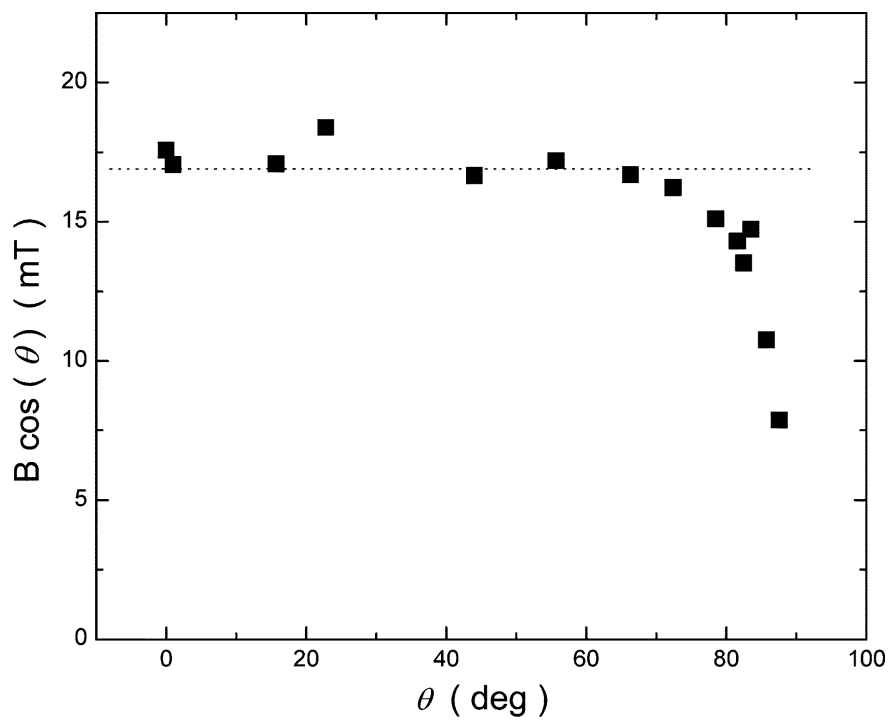

Fig. 6. Angular dependence of in-plane component of peak fields. Zero angle $\theta$ corresponds to magnetic field parallel to the film plane.

directions of the magnetic field with respect to the sample plane. The electric current has been applied along an edge of the square array. As can be seen from Fig. 6 we find that for angles smaller than $80^{\circ}$ from the film plane the position of peaks corresponds to an in-plane magnetic field of $\sim 17 \mathrm{mT}$ close to the value of in-plane coercive field. On the basis of numerical simulations and MFM imaging we relate the peaks to 90 degree switching of the onion state.

\section{CONCLUSION}

Magnetization reversal mechanisms and the impact of magnetic field direction are studied in square arrays of connected circular permalloy nanorings using MOKE, SEMPA, MFM, numerical simulations, and transport techniques. Angular dependences of in-plane coercive fields demonstrate significant variations reflecting impact of inter-ring connections. These connections should also affect switching in each particular ring and are detrimental for MRAM but can be considered for application in magnetic logic elements [10], [11]. Magnetoresistance acquires two pronounced peaks with a sign dependent on the current direction and position defined by an in-plane component of the field.

\section{ACKNOWLEDGMENT}

This work was supported in part by the U.K. Engineering and Physical Sciences Research Council and the USA National Science Foundation.

\section{REFERENCES}

[1] R. Skomski, J. Phys.: Cond. Matter, vol. 15, p. R841, 2003.

[2] A. Moser et al., J. Phys. D: Appl. Phys., vol. 35, p. R157, 2002.

[3] J. G. Zhu, Y. Zheng, and G. A. Prinz, J. Appl. Phys., vol. 87, p. 6668, 2000.

[4] J. Rothman et al., Phys. Rev. Lett., vol. 86, p. 1098, 2001.

[5] M. Klaui et al., Appl. Phys. Lett., vol. 81, p. 108, 2002.

[6] P. Vavassori et al., Phys. Rev. B, vol. 67, p. 134 429, 2003.

[7] M. R. Scheinfein, J. Unguris, M. H. Kelley, D. T. Pierce, and R. J. Celotta, Rev. Sci. Instrum, vol. 61, p. 2501, 1990.

[8] The Object Oriented Micromagnetic Framework. NIST, Gaithersburg, MD. [Online]. Available: http://math.nist.gov/oommf/

[9] A. V. Goncharov, A. A. Zhukov, V. V. Metlushko, G. Bordignon, H. Fangohr, G. Karapetrov, B. Ilic, and P. A. J. de Groot, J. Appl. Phys., vol. 99, 2006.

[10] U. Welp, V. K. Vlasko-Vlasov, G. W. Crabtree, J. Hiller, N. Zaluzec, V. Metlushko, and B. Ilic, J. Appl. Phys., vol. 93, p. 7056, 2003.

[11] R. P. Cowburn and M. E. Welland, Science, vol. 287, p. 5146, 2000.

[12] F. J. Kcastaňo et al., Phys. Rev. Lett., vol. 95, p. 137 201, 2005.

Manuscript received March 13, 2006 (e-mail: aaz@phys.soton.ac.uk). 